Gdańsk 2020, Nr. 42

https://doi.org/10.26881/sgg.2020.42.02

Michael Braun

(Konrad-Adenauer Stiftung Berlin / Universität zu Köln)

https://orcid.org/0000-0002-9349-3347

\title{
Werk ohne Autor? \\ Die Suche nach dem großen deutschen Roman in der Gegenwartsliteratur
}

Der „große deutsche Roman“, ein feuilletonistisches Schlagwort, ist ein Wunschbild, in dem seit den 1990er Jahren Erwartungen der Literaturkritik und der Anspruch auf nationale Repräsentanz zusammenfließen. Der Beitrag untersucht, wie es zu dieser Werkphantasie gekommen ist und was sie in der deutschen Gegenwartsliteratur angerichtet hat. Der ,große deutsche Roman', so die an Grass' Ein weites Feld und Pleschinskis Brabant (beide 1995) entwickelte These, ist ein Werk, das die Konzepte von Größe, von deutschen Traditionen und von Autorschaft in unterschiedlichen Modellen diskutiert.

Schlüsselwörter: Großer deutscher Roman, Wende, Identität, Geschichte, Erinnerungskultur

A literary work without an author? The search for the Great German Novel in contemporary literature. The Great German Novel, a catchy phrase of German feuilleton, is the ideal book of 'post-Wall'(postunification) literature that captures the universal quality of a reunified country and its national history. This essay deals with the American origins of this concept and its aftermaths in contemporary German literature. It is argued that the Great German Novel, if it is really such a novel, would retell stories of German grandiosity, German history, and be the product of a famous author. This idea of a grand narrative is discussed on the example of Grass's novel Ein weites Feld (Too Far Afield) and Hans Pleschinski's Brabant, both published in 1995 .

Keywords: Great German Novel, post-wall literature, identity, nation, memory culture

In der Tageszeitung Die Welt vom 10. Mai 2014 resümierte Wieland Freund unter dem Titel „Das Geheimnis des Einhorns“ die kurze Geschichte einer literarischen Chimäre, des großen deutschen Romans der Gegenwart. Nach der Wende 1989/90 hatte die Literaturkritik, so Freund,

beschlossen, die Entdeckung „des großen deutschen Romans“ zu verkünden. Doch dieses Ideal (oder Marketingkonzept) gibt es hierzulande nicht. Dem Publikum fehlt der heiße Wunsch, „den großen deutschen Roman“ zu lesen. Und den Autoren der Sportsgeist, ihn schreiben zu wollen. (Freund 2014) 
Hat es diesen Roman, um den sich Publikum, Literaturkritik und Kulturbetrieb streiten, wirklich nicht gegeben? Freunds Beispiel spricht nur zum Teil dafür. Der im Jahr 2000 erschienene, von der Frankfurter Allgemeinen Zeitung vorabgedruckte Roman Hampels Fluchten von Michael Kumpfmüller über einen Bettenverkäufer, der nach dem Krieg mit seinen Eltern aus Jena nach Regensburg zieht, seine Söhne Konrad und Walter auf die Namen der Gründerväter des geteilten Deutschlands tauft, 1962 zurück in den Osten geht und dort 1988 stirbt, wurde von den einen als der große deutsche Wenderoman gefeiert (Die Zeit, 17.8.2000; FAZ, 6.7.2000), von den anderen jedoch als komfortable Moritat, als „Nachtisch-Fassung der deutschen Geschichte" beargwöhnt (Neue Zürcher Zeitung, 26.9.2000; The Guardian, 16.11.2002): eine „Geschichte Gesamtdeutschlands von innen und unten gleichzeitig“ (Krekeler 2000).

Zwei Seiten hat auch der literaturwissenschaftliche Befund. Zwar sind allenthalben „Portalromane zur Vereinigungsproblematik" untersucht worden (Wehdeking 1995: 14), aber der "große Wenderoman“, der „die Sinn-Lücken des politischen Veränderungsschubs ausfüllen sollte", ist nicht darunter (Barner 2006: 965; vgl. Ledanff 2008).

Es geht mir hier weniger darum zu überprüfen, ob und inwiefern der große gesamtdeutsche Roman oder die Great German Novel, wie man sie in Anlehnung an die Great American Novel taufen könnte, für die Gegenwartsliteratur ein Kanonrezept liefert oder ob er ein komplexes Gattungshybrid ist - oder nur eine ambitionierte Erfindung der Literaturkritik. Wichtiger erscheint mir die Frage, was eigentlich hinter dieser Idee von dem großen deutschen Roman steckt. Was soll an ihm denn groß sein? Was hat das Deutsche, das er im Schilde führt, mit Mauerfall, Wende und deutscher Einheit, mit Staat und Nation zu tun? Und was oder wer könnte den großen deutschen Roman, wenn es ihn denn nicht gegeben haben sollte, verhindert haben?

Ich gehe in vier Schritten vor. Zunächst wird kurz die Wanderung des Begriffs der Great American Novel nach Deutschland verfolgt. Zweitens ist zu fragen, wer im Wunschkonzert des großen neuen Deutschlandepos sozusagen die Partitur liefert, den Grundtext, aus dem ein solches Werk überhaupt Stimme und Gestalt gewinnen kann. Damit hat vor allem das zu tun, womit ein Deutschland-Narrativ Anspruch auf Repräsentanz stellen kann: Das Erzählen von Geschichten im Rahmen staatsbegründender und staatsbildender Ereignisse samt ihrer Vorgeschichte - 1871 der Reichsgründung, 1919 der Weimarer Republik, 1949 der Bundesrepublik Deutschland und der DDR, 1989/90 des Mauerfalls und der Wiedervereinigung bürgt für eine Identität, die im Sinne einer Kulturnation, seltener patriotisch, ausgelegt wird. Der große deutsche Roman erzählt also, so die vorläufige Definition, eine Herkunfts- und Werdegeschichte von Deutschland und den Deutschen an einem markanten zeithistorischen Punkt (vgl. Peitsch 1993: 459-489). Dabei ist der Autor, wenn er diese Geschichte erzählt, nicht von dem Werk zu trennen. Ein dritter Abschnitt handelt daher, in gebotener Kürze, vom Anspruch auf Werk- und Autor-Repräsentanz in zwei 1995 erschienenen Romanen, Günter Grass' Ein weites Feld und Hans Pleschinskis Brabant, Werken, die ganz unterschiedlich in Struktur, Erzählweise, Intentionalität und Rezeption sind, die sich aber in einem Deutschlandbild treffen, das in der nationalliterarisch denkenden Zeit um 1900 - vor allem bei Fontane und Thomas Mann - späthumanistisch und europäisch geprägt ist. Das letzte Wort, hier zumindest, soll kein weiterer Kandidat auf die Great German Novel haben, sondern ein Film aus dem Jahr 2018, der schon im Titel Werk ohne Autor einen symbolischen Ausweg 
aus dem Finde-Dilemma vorschlägt und die literarische Repräsentanzfrage des wiedervereinigten Deutschland auf eine Nachbarkunst, das Bild, überträgt.

I.

Je näher man ein Wort anschaut, umso ferner blickt es zurück: Die Rede von der Great American Novel stammt aus der Gründungsphase des nach dem Bürgerkrieg wiedervereinigten Amerikas; der erste Beleg des Begriffs ist 1868 nachgewiesen. Er diente dazu, das Land als Buch zu lesen und die amerikanische Kultur von ihrer europäischen Einflussangst zu lösen.

It is that ideal book that captures some universal quality of American life and popular aspiration, and especially of quintessential patterns of American thought and speech at a particular time and place during the nation's history. For a truly timeless work, it would give an insight into enduring universalities of Americanness as perceived through a compelling story cast in idiomatic and ephemeral particulars. (García 2018)

Rasch wurde der Begriff dann zum Schlagwort, mit dem Romane unter nationalpatriotischem Segel auf Erfolgskurs gebracht wurden (Buell 2014). Bis heute hallt der Ruf nach der Great American Novel nach; ein Roman von Philip Roth aus dem Jahr 1973, der 2000 in deutscher Übersetzung erschien, führt genau diesen Titel.

Auffällig ist hier die Geburt des Begriffs aus dem Geist der gewissermaßen wiedervereinten amerikanischen Nation, die nach ihrer kulturellen Identität suchte und sie in einem Master-Narrativ zu finden hoffte. In Deutschland ist dieses Master-Narrativ für eine nationale Epopöe seit 1800 der Bildungsroman gewesen (Voßkamp 2009), der aber nur so lange von einer humanistischen Bildungsidee erzählen konnte, bis diese durch die Gewalt- und Kriegsgeschichte des 20. Jahrhunderts kontaminiert und in epische Bildungsparodien umgebogen wurde. Das exerziert geradezu vorbildlich Die Blechtrommel (1959), Günter Grass' Debütroman. Die Blechtrommel ist der erste große deutsche, ja gesamtdeutsche Roman der Nachkriegszeit. Hermann Kant polemisierte gegen die westdeutschen Kritiker, die den Autor 1960 als „,neuen Grimmelshausen“ vorstellten (Neumann 2009: 20), Hans Mayer lud ihn 1961 zu einer Lesung an die Universität Leipzig ein, 1987 erschien der Roman in einer Ost-Lizenzausgabe in der DDR (Hage 1987). Enzensberger bezeichnete den Roman als die „Lokomotive, die nach dem Krieg die deutsche Literatur an die Welt wieder herangeführt hat" (Neumann 2009: 74), und lobte schon bei seinem Erscheinen, dass der Protagonist „nicht nur seine eigene Geschichte“ erzähle, er sei „auch ein Mundstück der unsern" (Enzensberger 2009, 635). Die große Wirkung der Blechtrommel hat auch damit zu tun, dass Grass sich schelmisch und mit barocker Sprachkraft, und eben nicht ernst, predigend und trauervoll wie Frisch, Fried oder Christa Wolf (Schlaffer 2003: 149), der deutschen Sorgen annahm, von denen die Literatur in Deutschland nach Krieg und Holocaust geplagt war:

Seit 1945 hat die Herausforderung, vor der Deutsche standen, die über Deutsche schrieben, darin bestanden, das Trauma in Erinnerung zu verwandeln und die Gegenwart zu verstehen, indem sie die Vergangenheit betrauerten. Sie hatten zu zeigen, was es heißt, deutsch zu sein, indem sie Geschichten erzählten, die umfassend und tief genug waren, um das Unbeschreibliche in sich zu begreifen. (Boyle 2009: 220) 
Mit anderen Worten: Grass unterdrückte nicht die ,bösen 'Gedanken, die für große deutsche Romane nach 1945 oft die besseren waren, sondern inszenierte sie ,in munter-schwarzen Fabeln", so die Begründung der Schwedischen Akademie für den Literaturnobelpreis in ihrer Pressemitteilung (2009). Pikareskes Erzählen statt epischer Trauerschleier: Damit war Grass Roman nicht nur Prototyp für Schelmenromane, die nach der Wende von Thomas Brussig, Michael Kumpfmüller, Ingo Schulze und anderen geschrieben wurden. Mit dem kindlichen Helden, der hier für die Deutschen und zu den Deutschen spricht und der zu klein ist, um Verantwortung zu übernehmen, aber alt und frei genug, um Deutschland und seine zwielichtige Geschichte zu durchschauen, lieferte er auch eine neue Argumentationsfigur. Von einem wiedervereinigten Deutschland befürchtete er ungute Hegemonialphantasien und die Wiederkehr einer schlimmen Vergangenheit. In diesem Sinne votieren die Warnessays von Günter Grass gegen eine deutsche Einheit, die auf Kosten des jüdischen Opfergedächtnisses errungen würde.

Wenn man das weiterdenkt und die Teilung Deutschlands als möglichen Grund für das Ausbleiben des deutschen Großromans annimmt, dann muss das geeinte Deutschland einen staatstragenden deutschen Roman fast magisch anziehen. Schien von 1945 bis 1989 ein Roman ohne Staat nur als Sozialroman möglich, als Roman über die deutsche Gesellschaft und Kulturnation nach dem Modell von Thomas Manns Buddenbrooks (1901) (Boyle 2009, 169), so drängte die Staatseinheit seit 1989/90 umso stärker ins literarische Erzählen, noch bis in den jüngsten, mit dem Deutschen Buchpreis ausgezeichneten Deutschlandroman Stern 111 (2020) von Lutz Seiler:

Stern 111 ist ein politischer Roman, aber nicht, weil er von einer zentralen politischen Umbruchsepoche erzählt, sondern weil er den Glutkern alles Politischen, dessen Doppelnatur freilegt: die Einheit von poetischer Schwärmerei und Revolutionsmystik. Es ist ein Roman über die bohemistische Seite der politischen Romantik. Dichterische und politische Einbildungskraft leben vom selben Glauben an die Fantasie: dass die Welt neu benannt, verändert und also romantisiert werden kann. (Mangold 2020)

Wenn die erneuerte Nation ein Werk will, das ihr gültig und umfassend von ihrer neuen Identität erzählen kann, ist es freilich nützlich, zwischen Staat und Nation zu unterscheiden. Staatsbegründende Erzählungen - das können Romane sein, sind aber auch Gesetzestexte, Gründungslegenden, Erinnerungsrituale und Herkunftsmythen - sollen ja weniger den Staat als funktionierendes Ordnungs- und Rechtssystem tragen als vielmehr das nationale Selbstverständnis mit wenn nicht verbindlichen, so doch verbindenden Erzählungen, Symbolen und Ritualen ausstatten. Herfried Münkler macht als solche Staatsnarrative in Deutschland die „Europaerzählung von der Kriegsverhinderung“ und die viktimisierende Opfervorstellung sowie - als neue Narrative - die „Abendlanderzählung“ und die „Global-PlayerErzählung" deutlich (Münkler 2018), Aleida Assmann unterscheidet die klassischen deutschen Nationalerzählungen der Nachkriegszeit, nämlich Friedenssicherung und Rechtsstaatlichkeit, von den aktuellen europäischen Lektionen von Erinnerungskultur und Menschenrechten (Assmann 2018). 
II.

Die Frage nach der Größe des deutschen Romans steht im Zusammenhang einerseits mit der Theorie des großen Gesamtkunstwerks, andererseits mit dem Anspruch auf Größe der Autorschaft. Thomas Mann hat 1933 in seinem Essay Leiden und Größe Richard Wagners die Werkgröße an der „dramatische[n] Synthesis der Künste“ gemessen, „die nur als Ganzes, eben als Synthese, den Begriff des echten und legitimen Werks erfüllt" (Mann 1990: IX, 377), und er hat das, was an dieser Größe deutsch ist, in seinem Roman Doktor Faustus (1947) in das Schicksal eines Komponisten transformiert. Die Größe des Künstlers entspringt einem Werk, das gewaltig ist und das uns, in den Worten von Thomas Mann, ,als Phänomen deutschen und abendländischen Lebens" zu überwältigen vermag (Mann 1990: IX, 426). In seiner Schiller-Rede von 1955 hat Thomas Mann diese Idee von „Deutscher Größe“, die Schillers gleichnamiges Gedichtfragment als „sittliche Größe“ noch „in der Kultur" wohnen lässt (Schiller 1987: I, 473), allerdings vernünftigerweise wieder als Vormacht- und Übermachtphantasie relativiert.

Es ist kein Zufall, dass ausgerechnet die Autoren, die nach 1989/90 mit dem Anspruch auf Nationalrepräsentanz von Deutschland erzählten, Grass, Martin Walser, Christa Wolf, Botho Strauß, Peter Handke, in den Debatten der 1990er Jahre so oder so einen „Göttersturz“ erleiden mussten (Borchmeyer 2017: 919). Sie wurden einerseits aus unterschiedlichen Gründen als unzuverlässige Nationalautoren getadelt, andererseits aber zu „Sprechern der schweigenden Nation" geadelt, die sich zu sagen trauten, was - je nach demokratischer oder elitärer Auslegung - die Mehrheit des Volkes oder die Besten der Nation nicht auszusprechen wagten (Schmitz 2002: 338). Auf diese Weise konnte eine argumentierende statt erzählende Nationalrepräsentanz der Autorschaft in Essays und Reden das ersetzen (etwa bei Günter de Bruyn und Hans Magnus Enzensberger), was dem Roman an nationaler Größe fehlte.

Angefacht wurden die Diskussionen um den großen deutschen Roman vom Feuilleton. Es waren die Tages- und Wochenzeitungen, die auf der Bühne der wiedervereinigten Republik die Rollen für eine nationale Romanautorität zu- oder absprachen. Medienführend war Frank Schirrmacher, der 1989 als Nachfolger von Marcel Reich-Ranicki die Literaturredaktion der Frankfurter Allgemeinen Zeitung übernommen hatte und von 1994 bis zu seinem Tod 2015 als Mitherausgeber der FAZ die Debattenkultur steuerte. Sein Beitrag „Idyllen in der Wüste oder Das Versagen vor der Metropole", der in der Beilage der FAZ zur Herbstbuchmesse 1989 erschien, knüpfte den Anspruch des gesamtdeutschen Romans programmatisch an die Tradition der Metropolenliteratur und entdeckte, da er keinen Großstadtroman vom Stil Döblins ausfindig machen konnte, die Größe deutschen Erzählens in der Provinz, in der Idylle. Hinter dieser sofort heftig umstrittenen These steht die Idee von der Kulturnation, die im Kleinen überwintern konnte, wenn es in und von der Metropole nichts Großes zu erzählen gab. Solange sich die durch Zonengrenze und Mauer geteilte deutsche Nation durch eine gemeinsame Sprache und Literatur - gemäß der Konvergenzthese, dass es mehr Gemeinsamkeiten als Differenzen zwischen west- und ostdeutscher Literatur bis 1989 gab (Peitsch 2009: 35) - geeint wissen konnte, konnte ja wie gesagt der Roman ohne Staat und ohne Hauptstadt auskommen. Deshalb, so argumentiert Schirrmacher, blühte die Literatur aus der Provinz gerade in Gestalt von Novellen und Romanen auf, die die Deutschen zwar 
mit Motiven und Themen für ihre kulturelle Identität ausrüsten, aber keine nationale Einheitssymbolik stiften konnten: Heimatliteratur statt Metropolenroman.

Der zweite Essay von Schirrmacher, der nicht weniger Aufsehen erregte, erschien am 2. Oktober 1990, am Vorabend des ersten Tages der Deutschen Einheit. Unter der Überschrift „Abschied von der Literatur der Bundesrepublik“ hieß es:

Die Literatur der Bundesrepublik Deutschland wurde dreiundvierzig Jahre alt. Wie jener in der DDR steht auch ihr das Ende bevor. Nicht heute vielleicht, aber morgen. Dann wird ein großer Teil dieser Literatur Erinnerung an ein Land sein, das es nicht mehr gibt, und die Stimme einer Gesellschaft, deren Geräusche anders geworden sind. Da es wie ein Abschied zu Lebzeiten ist, vermeiden es die Betroffenen, von der Sache zu reden. Aber man sieht den Bruch. Was eben noch Gegenwart war, treibt davon. (Schirrmacher 1990)

Mit dem ersten Satz machte Schirrmacher eine neue Rechnung auf. 1947, also 43 Jahre zuvor, war das Gründungsdatum der Gruppe 47, einer literarisch und politisch einflussreichen Autorenvereinigung ohne Statuten. Die Gruppe 47 ,erfand' den deutschen Literaturbetrieb (Böttiger 2012: 15), ${ }^{1}$ ihre Autoren hatten die maßgeblichen Romane der deutschen Literatur geschrieben, Böll, Grass, Johnson und Walser vor allem. Schirrmachers Essay markiert ein Ende und eine Wende in der literaturkritischen Wahrnehmung der Gegenwartsliteratur, indem er ein Tor für Romane aufstößt, die vom und im wiedervereinigten Deutschland auf neue, ambitionierte Weise, also ,groß` erzählen können.

Und als ob die Literatur nur auf ein solches Startsignal gewartet hätte, erschienen 1995 gleich mehrere Romane, die als Anwärter auf den umfassenden deutschen Roman gehandelt wurden: Günter Grass' Ein weites Feld, Thomas Hettches Nox, Erich Loests Nikolaikirche, Thomas Brussigs Helden wie wir, Reinhard Jirgls Abschied von den Feinden, Bernhard Schlinks Der Vorleser und Hans Pleschinskis Brabant. Hettches und Brussigs Romane erzählen deutsche Geschichte, während sie sich ereignet; sie sind burleske Präsenzliteratur im Angesicht von Mauerfall und Wende. Loests Nikolaikirche ergänzt die unmittelbare Vorgeschichte vor allem in Leipzig, Jirgls Roman fügt die Nachkriegsgeschichte von Flucht und Vertreibung hinzu. Schlinks Roman verbindet die Nationalnarrative der Erinnerungskultur und der Kriegsverhinderung mit der zentralen Frage, ob und was man aus der deutschen Geschichte noch lernen kann.

III.

Günter Grass' Roman ist der wohl am heftigsten umstrittene Deutschlandroman nach der Wiedervereinigung. Umstritten war weniger der Inhalt, der die deutsche Gegenwart in die Geschichte des 19. Jahrhunderts vertiefte, sondern genau dieser Anspruch des Romans, die deutsche Geschichte durch Tiefe groß zu machen. Das Nachrichtenmagazin Der Spiegel vom 21. August 1995 veröffentlichte in diesem Sinne einen bitterbösen Offenen Brief von Marcel Reich-Ranicki. Der Literaturkritiker las seinem besten Feind (vgl. Weidermann 2019)

1 Die Gruppe 47 stiftete das Narrativ vom Ursprung der Nachkriegsliteratur im surrealistisch eingefärbten Kahlschlag, das nicht totzukriegende ,Fräulein Kafka', die Eventisierung der Lesung zum Betriebsspektakel, die Prädominanz der Kritik, das Fallbeil über Autorenkarrieren und die Kunst der medialen Selbstinszenierung. 
die Leviten, „ganz und gar mißraten“ sei der Roman, weil der ,größenwahnsinnige “ Autor seinem selbstgestellten Auftrag, Deutschland eine zeitgerechte Geschichte zu geben, eben nicht gerecht geworden sei. Reich-Ranickis Polemik wurde durch das Spiegel-Cover verschärft, das eine mit dem Kritiker nach dessen eigenen Worten nicht abgesprochene Montage zeigte: Reich-Ranicki verreißt den Roman nicht nur, er zerreißt ihn. Das Entzweireißen erinnert an Rembrandts Bild vom Zerschmettern der Gesetzestafeln (1659) und zugleich an die episch zerrissene Einheit Deutschlands.

Daniel Kehlmann hat sich in einem Nachwort zur Neuausgabe von Ein weites Feld im Fontane-Gedenkjahr 2019 gegen die literaturkritische Verengung des Romans auf die Gegenwart gewandt. Es ging Grass, so schreibt er, ,eben nicht um die große Gegenwartsdiagnose, sondern um die Durchdringung der Gegenwart und ihrer Vorbedingungen mit den Mitteln souveräner Ästhetik“" (Kehlmann 2019: 7). Literarische Größe in Grass` Roman ist die Größe anderer Autoren, auf die er sich bezieht, von Theodor Fontane als kritischem Begleiter der Reichsgründungsjahre im 19. Jahrhundert und von Hans Joachim Schädlich als schriftstellerischem Zeitzeugen des geteilten und des geeinten Deutschlands. Fonty heißt eine Romanfigur, Hoftaller heißt, im Silbentausch mit Schädlichs Romanfigur Tallhover, ihr Begleiter. Es ist also eine geborgte Repräsentanz, eine Größe auf den Schultern von Riesen, eine Grandiositätsphantasie. Im Weiten Feld wie in der Blechtrommel bannt eine allmächtige Figurenstimme Angst und Scham und ermächtigt eine moralische Instanz zum Erzählen (Morsbach 2006: 166).

Diese Überlegenheitsphantasie im Werk wird in zwei Kapiteln des Romans Ein weites Feld deutlich. „Vor das Denkmal gestellt" und „Vom Denkmal herab gesprochen“ (Kap. 28 und 29) erzählen, wie die Hauptfiguren, der fontanefanatische Theo Wuttke, genannt Fonty, und sein mephistophelischer Begleiter Hoftaller, also die umgedrehte Figur des ewigen Spitzels aus Schädlichs Roman, das Fontane-Denkmal in Neuruppin besuchen. Hoftaller bringt Wuttke mit seinem Insiderwissen über die Stasi-Verwicklung von dessen Sohn erpresserisch dazu, das Denkmal zu erklettern und sich neben die Bronzefigur zu setzen. Die Romanfigur neben ihrem Vorbild, das verdoppelte Denkmal: Auch das ist eine Größenimitation, die jedoch ins rechte Maß zum Original gerückt wird: „[...] auch Fonty spürte das Überlebensgroße, nun da er Arm an Arm saß. Neben ihm dominierte das Original. Zwar mangelte es nicht an Ähnlichkeit, doch wirkte die verkleinerte Ausgabe wie ein geschrumpftes Modell“ (Grass 2019: 578). Und wie „zum Beweis“ - was im Roman als metadiegetischer Autor-Kommentar lesbar ist - kommt der Autor Günter Grass selbst „, [s]törend und doch wie selbstverständlich dem erzählten Verlauf dazwischen“ (Grass 2019: 580). Da nähert sich nämlich, „aus den Tiefen der Parkanlage“, ein schon älteres Paar mit Photoapparat dem Denkmal, er „bullig gedrungen“, „,mit Baskenmütze“ und verräterischem „Interesse an Details“(Grass 2019: 579). Kein Zweifel, das kann nur eine Spiegelfigur von Grass sein, und diese Figur sagt dann zu seiner Frau: „Guck mal, neben unserem Freund ist viel Platz“ (Grass 2019: 579f.). So hat sich Grass hier selbst als Autor ins Werk gesetzt, um das Große an der Größenphantasie zu symbolisieren.

Eine ähnlich nationalcodierte Szene findet sich in Hans Pleschinskis Roman Brabant. Darin geht es um die Atlantikfahrt einer multinationalen europäischen Kulturgesellschaft, die in den 1990er Jahren mit einem Warnschuss in Washington D. C. gegen die Errichtung eines Disney-Parks in Rom protestieren will. Es ist ein Narrenschiff und zugleich ein Transportmittel für nahezu alle modernen Europa-Diskurse, den späthumanistischen wie 
den postkolonialistischen, den universalistischen wie den nationalkulturellen, den proeuropäischen wie den antiamerikanischen. In dem Kapitel „Omaha-Beach“, das den Tag der amerikanischen Landung in der Normandie 1944 umkehrt zur Invasion von empörten Kultureuropäern, finden wir ein „Testament an die Deutschen“ (Pleschinski 2004: 553). Es ist von dem Altertumswissenschaftler Erich Müller geschrieben, der die Deutschen als Romanfigur vertritt. Müllers „Testament“ ist, in der Tradition von Hölderlins Vaterlandsschelte im Hyperion (1797/99) und von Thomas Manns Radioansprachen an Deutsche Hörer (1940-1945), ein Appell an Frieden und Völkerliebe, zugleich aber auch das Dokument einer Suche nach dem, was die Deutschen denn an Großem der Welt hinterlassen können:

Die wirklichen Künstler haben nie deutsche Kunst erschaffen, sondern Kunst für sich selbst, manchmal für andere. Die Bindungen der Künstler durchdringen alle Zeiten und Orte. Der Goldgrund hinter gotischen Madonnen stammt aus Byzanz, alle Säulen sind griechisch. In welchem Land würden Bilder, Skulpturen und Klänge aus gleich welcher Seele ihren Ausdruck verlieren?

Die Künstler, liebe Deutsche, sind eure Weltheimat. (Pleschinski 2004: 557)

Pleschinski lässt seine Figur dagegen protestieren, dass Krieg und Holocaust „Maßstab“ für das Erbe der Deutschen sind, und daran erinnern, dass Deutschland ein „Handelsland“ war, Kaiser mit „Seelenadel“ hatte und frühe Demokraten, die ihren „Freiheitsbaum schon 1792 in Mainz pflanzten“" (Pleschinski 2004: 556). Groß am Deutschlandbild sind hier die positiven Traditionen von Republik und Demokratie, von Fortschritt und Freiheit, Recht und guter Ordnung. ${ }^{2}$

\section{IV.}

Grass und Pleschinski entwerfen unterschiedliche, aber auch vergleichbare Deutschlandnarrative. Um Deutschland im Roman nicht zu groß zu erzählen, wählen beide die unbescholtene Gattung des pikaresken Romans und ein Erzählerensemble, das bei Grass aus den Mitarbeitern des Fontane-Archivs und aus dem uneinigen Duo Fonty - Hoftaller (vgl. Scheitler 2008), bei Pleschinski aus europäischen Kulturenthusiasten mit 29 Staatsangehörigkeiten besteht. Grass lässt in seinem Roman mehr das Staatsnarrativ von der Kriegsabwendung gelten, das sich in der Erinnerungskultur nach 1945 als Wiederholungsverbot, als „Nie wieder“-Appell Geltung verschafft hat. Er inszeniert eine moralisch-pädagogische Erinnerungskultur, Pleschinski lässt seine Figuren mehr die europäischen Lektionen von „Abendlanderzählung“ und Menschenrechten beherzigen: hier eher das positive, dort eher das negative Vermächtnis der deutschen Geschichte. Beide Romane vertreten durchaus den Anspruch, für ein gesamtes Deutschland zu sprechen, das sich seiner Geschichte bewusst ist und seine Identität, national wie auch europäisch, mit einem großen Reichtum an Symbolen und Emblemen erzählen kann.

Und was ist mit dem großen deutschen Erzähler? Grass hat diesen Anspruch als Gewissen der Nation, als Mahner und Warnprophet seit seinem Debüt immer wieder behauptet, Pleschinski hat diesen Titel mit „Eleganz und Eigensinn“ (Wolfinger/Schütz 2019) umschifft, aber wiederum ähnlich wie Grass die Nationalrepräsentanz der Autorschaft auf Leitfiguren

2 Vgl. auch die Essays von Hans Pleschinski über byzantinische Leitfiguren der deutschen und europäischen Kultur in: Byzantiner und andere Falschmünzer. ElfLichter im Dunkel. Frankfurt a.M.: Schöffling 1997. 
der deutschen Literatur übertragen, auf Thomas Mann (in dem Roman Königsallee, 2013) und auf Gerhart Hauptmann (in dem Roman Wiesenstein, 2018).

Vielleicht aber kann man auch auf den Erzähler verzichten, auf den der deutsche Wenderoman immer noch wartet (Bohrer 1995). Der Film Werk ohne Autor von Florian Henckel von Donnersmarck, der bereits mit seinem Stasi-Film Das Leben der Anderen (2006) ein sehr kritisches, aber auch differenziertes Bild von Künstlerphantasie und Staatsmacht entworfen hat, inszeniert einen deutschen Lebenslauf, der durch zwei Diktaturen in die wiedervereinigte Republik verläuft und statt des Künstlers die Kunst für Deutschland und die Deutschen sprechen lässt (vgl. Braun 2019). Orientierungsfigur des Biopics ist Gerhard Richter, der im Film Kurt Barnert heißt. Er wächst während der Hitlerzeit in der Nähe von Dresden auf, studiert in der DDR an der Dresdner Kunstakademie und wird zum vielgefragten Staatskünstler, geht dann aber, vor dem Mauerbau, nach Düsseldorf und entwickelt hier die eigentümliche Form des Fotorealismus, die sein erstes Markenzeichen wird. Parallel dazu erzählt der Film die Geschichte von Kurts Tante, die im nationalsozialistischen Euthanasie-Programm zu Tode kommt, und zwar unter der Verantwortung von Kurts Schwiegervater, dem Medizinprofessor Kurt Seeband. Die Figuren haben reale Vorbilder in der Geschichte - und Nachbilder in der Kunst, denn Gerhard Richter hat sowohl seine Tante (Tante Marianne, 1965) wie auch seinen Schwiegervater Heinrich Eufinger porträtiert (Familie am Meer, 1964). Diese Täter-OpferKonstellation, die dem Maler selbst, als er diese Bilder malte, noch nicht bewusst war, ${ }^{3}$ wird in einer entscheidenden Szene des Films visualisiert. Im Düsseldorfer Atelier werden die Fotoporträts von Barnerts Schwiegervater und seiner Tante ineinandergeblendet, das Foto des Täters wird mithilfe eines Episkops auf das Porträt von dessen Opfer geworfen. Das geschieht mittels eines Windstoßes, der die Fensterläden zuschlagen lässt und so das Lichtbild des Täters sichtbar macht. Es ist der Wind der Geschichte, könnte man sagen, oder besser: der Eigensinn des Kunstwerks im Augenblick seiner Entstehung, der eine zentrale Erinnerung, die massiv und repräsentativ im deutschen Gedächtnis steht, mobilisiert. Der Film erzählt hier mit Bildern und in Bildern, er beobachtet die Bilder der deutschen Täter-Opfer-Verwandtschaft. Die erzählende Kamera lässt das Kunstwerk auf diese Weise mehr wissen als den Künstler. Das Medium selbst, die Kunst, ist es, die eine deutsche Geschichte erzählt und in diesem künstlerischen Erinnerungsbild nicht unbedingt groß, aber repräsentativ sein kann für andere Täter-Opfer-Konstellationen.

\section{Literatur}

Assmann, Aleida (2018): Der europäische Traum. Vier Lehren aus der Geschichte. München: C. H. Beck. Barner, Wilfried u. a. (Hg.) (2014): Geschichte der deutschen Literatur von 1945 bis zur Gegenwart (2., aktualisierte und erw. Aufl.). München: C. H. Beck.

Böttiger, Helmut (2012): Die Gruppe 47. Als die deutsche Literatur Geschichte schrieb. München: Deutsche Verlags-Anstalt.

3 Das erfolgte erst durch Jürgen Schreibers Dokumentarrecherche Ein Maler aus Deutschland. Gerhard Richter (2005), auf die der Regisseur des Films zurückgegriffen hat. 
Bohrer, Karl-Heinz (1995): Erinnerung an Kriterien. Vom Warten auf den deutschen Zeitroman. In: Merkur. Deutsche Zeitschrift für europäisches Denken. 49/11, 1055-1061.

Borchmeyer, Dieter (2018): Was ist deutsch? Die Suche einer Nation nach sich selbst. Berlin: Rowohlt. Boyle, Nicholas (2009): Kleine deutsche Literaturgeschichte. Aus dem Englischen übersetzt von Martin Pfeiffer. München: C. H. Beck.

Braun, Michael (2019): Erinnerung und Wahrheit im Bild. Medienobservationen in Florian Henckel von Donnersmarcks Werk obne Autor (2018). In: medienobservationen, 15.10. Online: https:// www.medienobservationen.de/2019/1015-braun/ (Abfrage am 22.01.2020).

Buell, Lawrence (2014): The Dream of the Great American Novel. Cambridge/Ma., London: Harvard University Press.

Enzensberger, Hans Magnus (2009): Wilhelm Meister, auf Blech getrommelt (1959). In: Ders.: Scharmützel und Scholien. Über Literatur. Frankfurt a.M.: Suhrkamp, 629-635.

Freund, Wieland (2014): Das Geheimnis des Einhorns. In: Die Welt, 10.05.

García, Manuel (2018): The Ultimate Great American Novel. In: CounterPunch, 06.09.

Grass, Günter (2019): Ein weites Feld. Roman. Hg. von Dieter Stolz und Werner Frizen. Göttingen: Steidl.

Hage, Volker (1987): Kein Respekt. In: Der Spiegel, 18.09.

Kehlmann, Daniel (2019): „Ihm sind die Jahrhunderte durchlässig gewesen“. Ein Vorwort. In: Günter Grass: Ein weites Feld. Roman. Hg. von Dieter Stolz und Werner Frizen. Göttingen: Steidl, 5-9.

Krekeler, Elmar (2000): Das Buch der Woche: Dann geh doch nach drüben. In: Die Welt, 19.08.

Ledanff, Susanne (2008): Die Suche nach dem „Wenderoman“ - zu einigen Aspekten der literarischen Reaktionen auf Mauerfall und deutsche Einheit in den Jahren 1995 und 1996. In: http://www. dickinson.edu/glossen/heft2/wende.html (Abfrage am 14.01.2020).

Mangold, Ijoma (2020): „Die Häuser denen, die drin wohnen“. Lutz Seilers neuer Roman Stern 111 erzählt von 1989 - aber in Wahrheit von Glanz und Elend der politischen Romantik. In: Die Zeit, 26.02 .

Mann, Thomas (1990): Gesammelte Werke in 14 Bänden. Frankfurt a.M.: S. Fischer.

Morsbach, Petra (2006): Warum Fräulein Laura freundlich war. Über die Wabrheit des Erzählens. München, Zürich: Piper.

Münkler, Herfried (2018): Auf der Suche nach einer neuen Europaerzählung. In: Grit Straßenberger und Felix Wassermann (Hg.): Staatserzählungen. Die Deutschen und ibre politische Ordnung. Berlin: Rowohlt, 169-196.

Neumann, Uwe (2009): Trommelwirbel. Fünfzig Jahre „Die Blechtrommel“. Göttingen: Steidl.

Peitsch, Helmut (1993): „Antipoden“ im „Gewissen der Nation“? Günter Grass“ und Martin Walsers „deutsche Fragen“. In: Helmut Scheuer (Hg.): Dichter und ihre Nation. Frankfurt a.M.: Suhrkamp.

Peisch, Helmut (2009): Nachkriegsliteratur 1945-1989. Göttingen: Vandenhoeck \& Ruprecht.

Pleschinski, Hans (2004): Brabant. Roman zur See. Revidierte Neuausgabe. München: dtv.

Pressemitteilung (2009): Pressemitteilung der Ständigen Sekretärs der Schwedischen Akademie, 30.09.2009: https://www.nobelprize.org/prizes/literature/1999/7856-gunter-grass-1999-3/ (Abfrage am 14.01.2020).

Reich-Ranicki, Marcel (1995): ... und es muß gesagt werden. Offener Brief an Günter Grass. In: Der Spiegel, 21.08. 
Scheitler, Irmgard (2008): Günter Grass: Ein weites Feld. In: Sabine Schneider (Hg.): Lektüren für das 21. Jahrhundert. Klassiker und Bestseller der deutschen Literatur von 1900 bis heute. Würzburg: Königshausen \& Neumann, 141-158.

Schiller, Friedrich (1982): Sämtliche Werke in fünfBänden. Hg. von Herbert G. Göpfert und Gerhard Fricke. Darmstadt: Wissenschaftliche Buchgesellschaft [= Lizenzausgabe der Hanser-Ausgabe]. Schirrmacher, Frank (1989): Idyllen in der Wüste oder Das Versagen vor der Metropole. In: Frankfurter Allgemeine Zeitung, 10.10. (Wiederabdruck in: Andrea Köhler [Hg.]: Maulhelden und Königskinder. Zur Debatte über die deutschsprachige Gegenwartsliteratur. Leipzig: Reclam, 1998, 15-27).

Schirrmacher, Frank (1990): Abschied von der Literatur der Bundesrepublik. Neue Pässe, neue Identitäten, neue Lebensläufe. Über die Kündigung einiger Mythen des westdeutschen Bewusstseins. In: Frankfurter Allgemeine Zeitung, 02.10.

Schlaffer, Heinz (2002): Die kurze Geschichte der deutschen Literatur. München: Hanser.

Schmitz, Walter (2002): Ist der ,klassische Nationalautor ' bei den Deutschen ausgestorben? Günter Grass und Martin Walser im Wandel des literarischen Feldes. In: Marek Zybura unter Mitwirkung von Kazimierz Wóicicki (Hg.): Geist und Macht. Schriftsteller und Staat im Mitteleuropa des „kurzen Jahrhunderts"1914-1991. Dresden: Thelem, 337-362.

Voßkamp, Wilhelm (2009): Der Roman eines Lebens. Die Aktualität unserer Bildung und ihre Geschichte im Bildungsroman. Berlin: berlin university press.

Wehdeking, Volker (1995): Die deutsche Einheit und die Schriftsteller. Literarische Verarbeitung der Wende seit 1989. Stuttgart, Berlin, Köln: Kohlhammer.

Weidermann, Volker (2019): Das Duell. Die Geschichte von Günter Grass und Marcel Reich-Ranicki. Köln: Kiepenheuer\&Witsch.

Wolfinger, Kai und Laura Schütz (Hg.) (2019): Eleganz und Eigensinn. Studien zum Werk von Hans Pleschinski. Würzburg: Königshausen \& Neumann. 